\title{
On Harmonic Index and Diameter of Graphs*
}

\author{
Jianxi Liu \\ School of Informatics, Guangdong University of Foreign Studies, Guangzhou, China \\ Email: liujianxi2001@gmail.com, ljx@oamail.gdufs.edu.cn
}

Received June 12, 2013; revised July 15, 2013; accepted August 5, 2013

Copyright (C) 2013 Jianxi Liu. This is an open access article distributed under the Creative Commons Attribution License, which permits unrestricted use, distribution, and reproduction in any medium, provided the original work is properly cited.

\begin{abstract}
The harmonic index of a graph $G$ is defined as $H(G)=\sum_{u v \in E(G)} \frac{2}{d(u)+d(v)}$, where $d(u)$ denotes the degree of a vertex $u$ in $G$. It has been found that the harmonic index correlates well with the Randi $c^{\prime}$ index and with the $\pi$-electronic energy of benzenoid hydrocarbons. In this work, we give several relations between the harmonic index and diameter of graphs.
\end{abstract}

Keywords: Harmonic Index; Diameter

\section{Introduction}

All graphs considered in the following will be simple. Let $G$ be a graph with vertex set $V(G)$ and edge set $E(G)$. The order of graph $G$ is the number of its vertices. The leaf of a graph is a vertex with degree one. For undefined terminology and notations we refer the reader to [1]. For a graph $G$, the harmonic index $H(G)$ is defined as

$$
H(G)=\sum_{u v \in E(G)} \frac{2}{d(u)+d(v)} .
$$

It has been found that the harmonic index correlates well with the Randic index [2,3] and the $\pi$-electronic energy of benzenoid hydrocarbons [4,5]. Favaron et al. [6] considered the relation between harmonic index and the eigenvalues of graphs. Zhong [7] found the minimum and maximum values of the harmonic index for connected graphs and trees, and characterized the corresponding extremal graphs. Recently, Wu et al. [8] gave the minimum value of the Harmonic index among the graphs with the minimum degree at least two. In this work, we shall give some relations between the harmonic index and diameter of graphs.

\section{Main Results}

First, we shall give two sharp upper bounds of the

*Research supported by the National Natural Science Foundation of China (No.11101097) and Foundation for Distinguished Young Talents in HigherEducation of Guangdong, China (No.LYM11061). relationship involving the harmonic index and diameter of connected graphs. In [7], Zhong gave the following result:

Lemma 2.1 ([7]) Let $G$ be a graph with order $n$, then $H(G) \leq \frac{n}{2}$, where the equality holds if and only if $G$ is a regular graph.

Since the complete graph $K_{n}$ is a regular graph with diameter one, we have:

Theorem 2.2 Let $G$ be a connected graph with order $n(\geq 4)$ diameter $D(T)$, then

$$
H(T)-D(T) \leq \frac{n}{2}-1, \frac{H(T)}{D(T)} \leq \frac{n}{2}
$$

where equalities hold if and only if $G$ is the complete graph $K_{n}$.

An edge $x_{1} x_{2}$ is called local maximum if its weight $\frac{2}{d\left(x_{1}\right)+d\left(x_{2}\right)}$ is maximum in its neighborhood, i.e.,

$$
\frac{2}{d\left(x_{1}\right)+d\left(x_{2}\right)} \geq \frac{2}{d\left(x_{i}\right)+d(u)}
$$

for any edge $x_{i} u$ for $i=1,2$.

Lemma 2.3 Let $x_{1} x_{2}$ be a local maximum edge in graph $G$, then

$$
H(G)-H\left(G-x_{1} x_{2}\right)>0 .
$$

Proof. We have 


$$
\begin{aligned}
& H(G)-H\left(G-x_{1} x_{2}\right)=\frac{2}{d\left(x_{1}\right)+d\left(x_{2}\right)}+\sum_{u \in N\left(x_{1}\right) \backslash\left\{x_{2}\right\}}\left(\frac{2}{d\left(x_{1}\right)+d(u)}-\frac{2}{d\left(x_{1}\right)+d(u)-1}\right) \\
& +\sum_{v \in N\left(x_{2}\right) \backslash\left\{x_{1}\right\}}\left(\frac{2}{d\left(x_{2}\right)+d(v)}-\frac{2}{d\left(x_{2}\right)+d(v)-1}\right) \geq \frac{2}{d\left(x_{1}\right)+d\left(x_{2}\right)}+\left(d\left(x_{1}\right)-1\right)\left(\frac{2}{d\left(x_{1}\right)+d\left(x_{2}\right)}-\frac{2}{d\left(x_{1}\right)+d\left(x_{2}\right)-1}\right) \\
& +\left(d\left(x_{2}\right)-1\right)\left(\frac{2}{d\left(x_{1}\right)+d\left(x_{2}\right)}-\frac{2}{d\left(x_{1}\right)+d\left(x_{2}\right)-1}\right)=\frac{2}{\left(d\left(x_{1}\right)+d\left(x_{2}\right)-1\right)\left(d\left(x_{1}\right)+d\left(x_{2}\right)\right)}>0,
\end{aligned}
$$

where $N\left(x_{i}\right)$ denotes the vertex set adjoining to $x_{i}$ for $i=1,2$.

If $x_{1} x_{2}$ is a leaf of $G$, i.e., at least one of $\left\{x_{1}, x_{2}\right\}$ has degree one, we can see that it is a local maximum edge. Thus, by Lemma 2.3,

Corollary 2.4 If $x_{1} x_{2}$ is a leaf in graph $G$, then

$$
H(G)-H\left(G-x_{1} x_{2}\right)>0 .
$$

Theorem 2.5 Let $T$ be a tree with order $n(\geq 4)$ diameter $D(T)$, then

$$
H(T)-D(T) \geq \frac{5}{6}-\frac{n}{2}, \frac{H(T)}{D(T)} \geq \frac{1}{2}+\frac{1}{3(n-1)}
$$

where equalities hold if and only if $T$ is a path $P_{n}$.

Proof. If $T$ is a path, we have $H(T)=\frac{n}{2}-\frac{1}{6}$ and $D(T)=n-1$. It is obvious that both equalities hold. Now we assume that $T$ is not a path, then $D(T) \leq n-2$ and there are at least three pendent vertices in $T$. Assume $P=u_{0} u_{1} \cdots u_{D}$ be the longest path in $T$. Then at least one pendent vertex, say $v_{1}$, is not contained in $P$. Now we start an operation on $T$, i.e., we continually delete pendent vertices which are not contained in $P$ until the resulting tree is $P$. Assume $v_{1}, \cdots, v_{k}$ are the vertices in the order they were deleted, we have

$$
H(T)>H\left(T-v_{1}\right)>\cdots>H\left(T-\bigcup_{i=1}^{k} v_{i}\right)=H(P)=\frac{D}{2}+\frac{1}{3}
$$

by Corollary 2.4 and

$$
D(T)=D\left(T-v_{1}\right)=\cdots=D\left(T-\bigcup_{i=1}^{k} v_{i}\right)=D \text {. Thus, we }
$$

have

$$
\begin{aligned}
& H(T)-D(T)>H(P)-D(P) \\
& \geq \frac{5}{6}-\frac{D+1}{2} \geq \frac{5}{6}-\frac{n-1}{2}>\frac{5}{6}-\frac{n}{2}
\end{aligned}
$$

and

$$
\frac{H(T)}{D(T)}>\frac{H(P)}{D(P)}=\frac{\frac{D+1}{2}-\frac{5}{6}}{D}>\frac{\frac{n}{2}-\frac{1}{6}}{n-1} .
$$

This result seems true for any connected graph with order $n$ and we propose as a conjecture as follows:

Conjecture 2.6 Let $G$ be a connected graph with order $n(\geq 4)$ diameter $D(G)$, then

$$
H(G)-D(G) \geq \frac{5}{6}-\frac{n}{2}, \frac{H(G)}{D(G)} \geq \frac{1}{2}+\frac{1}{3(n-1)} .
$$

\section{REFERENCES}

[1] J. A. Bondy and U. S. R. Murty, "Graph Theory," Springer, Berlin, 2008.

[2] X. Li, I. Gutman, "Mathematical Aspects of Randić-Type Molecular Structure Descriptors," Mathematical Chemistry Monographs No.1, University of Kragujevac, 2006.

[3] X. Li and Y. T. Shi, "A Survey on the Randić Index," Communications in Mathematical and in Computer Chemistry, Vol. 59, No. 1, 2008, pp. 127-156.

[4] B. Lučić, N. Trinajstić and B. Zhou, "Comparison between the Sum-Connectivity Index and Product- Connectivity Index for Benzenoid Hydrocarbons," Chemical Physics Letters, Vol. 475, No. 1-3, 2009, pp. 146-148. doi:10.1016/j.cplett.2009.05.022

[5] B. Lučić, S. Nikolić, N. Trinajstić, B. Zhou and S. I. Turk, "Sum-Connectivity Index," In: I. Gutman and B. Furtula, Ed., Novel Molecular Structure Descriptors-Theory and Applications I, University of Kragujevac, Kragujevac, 2010, pp. 101-136.

[6] O. Favaron, M. Mahó and J. F. Saclé, "Some Eigenvalue Properties in Graphs (Conjectures of Graffiti-II)," Discrete Mathematics, Vol. 111 No. 1-3, 1993, pp. 197-220. doi:10.1016/0012-365X(93)90156-N

[7] L. Zhong, "The Harmonic Index for Graphs," Applied Mathematics Letters, Vol. 25, No. 3, 2012, pp. 561-566. doi:10.1016/j.aml.2011.09.059

[8] R. Wu, Z. Tang and H. Deng, "A Lower Bound for the Harmonic Index of a Graph with Minimum Degree at Least Two," Filomat, Vol. 27, No. 1, 2013, pp. 51-55. 